\title{
Theoretical Modeling Suggests that Synergy May Result from Combined Use of Two Biocontrol Agents for Controlling Foliar Pathogens Under Spatial Heterogeneous Conditions
}

\author{
X.-M. Xu and M. J. Jeger
}

\begin{abstract}
First author: State Key Laboratory of Crop Stress Biology in Arid Areas and College of Plant Protection, Northwest A\&F University, Yangling, Shaanxi 712100, China, and Pest and Pathogen Ecology for Sustainable Crop Management, East Malling Research, New Road, East Malling, ME19 6BJ, UK; second author: Division of Ecology and Evolution and Centre for Environmental Policy, Imperial College London, Silwood Park campus, Ascot, SL5 7PY, UK.
\end{abstract} Accepted for publication 14 March 2013.

\begin{abstract}
Xu, X.-M., and Jeger, M. J. 2013. Theoretical modeling suggests that synergy may result from combined use of two biocontrol agents for controlling foliar pathogens under spatial heterogeneous conditions. Phytopathology 103:768-775.

There has been a trend for combined use of several biocontrol agents (BCAs) with an expectation of synergistic interactions among BCAs. However, previous modeling studies suggested that, under homogeneous and temporal-fluctuating conditions, combined use of two BCAs, in most cases, only results in efficacies similar to the more efficacious one used alone; a result consistent with published experimental data. The present modeling study investigated whether combined use of two mycoparasitic BCAs, two competitive BCAs, or a mycoparasitic and a competitive BCA leads to synergistic interactions under spatially heterogeneous conditions. In the model, there were two patches with varying relative sizes and two BCAs differentially adapted to the two patches. Within the range of model parameter values considered, combined use of two BCAs is more effec-
\end{abstract}

ABSTRACT tive than the more efficacious BCA used alone in $72 \%$ of the simulated cases. There was also a considerable proportion $(\approx 21 \%)$ of model simulations in which combined use of two BCAs led to synergy (i.e., efficacy was greater than expected under the assumption of Bliss independence, especially when each of the two BCAs can only survive in one [different] patch). Combined use of a mycoparasitic BCA with a competitive one is more likely to result in synergy than the other two BCA combinations. When biocontrol activities of individual BCAs are low or moderate, biocontrol efficacy arising from combined use of two BCAs does not depend greatly on biocontrol mechanisms. However, for high BCA activities, combined use with at least one competitive BCA resulted in better control than combined use of two mycoparasitic BCAs. The present modeling study emphasized the need for understanding the degree of spatial patchiness and quantitative relationships between biocontrol activities and external conditions in order to apply commercial BCAs effectively.

Additional keywords: spatial heterogeneity.
Effective use of biocontrol agents (BCAs) is a potentially important component of sustainable agriculture. Because of the complex ecological processes involved, success in biocontrol of plant diseases in field crops has been limited (4); most successes being achieved in greenhouse cultivation $(6,18)$. To improve control efficacies, there has recently been increasing interest in studying experimental efficacy arising from combined use of BCAs. It has been suggested that combined use may have two potential benefits: that is, synergy in control efficacies and reduced variability in the efficacy achieved. However, a recent review of published experimental evidence on the combined use of BCAs showed that, contrary to the frequent claim of synergy, most published experimental data instead showed antagonistic interactions among BCAs (23). There is, as yet, insufficient experimental evidence to either support or refute the second advantage claimed for the combined use of BCAs (i.e., reduced variability) (9).

Modeling results from a mean-field deterministic model for biocontrol of foliar diseases suggest that combining mechanisms within a single BCA is generally the most effective in reducing disease development (26), and that two BCAs with a single but different mechanism would rarely combine synergistically (24), in

Corresponding author: X.-M. Xu; E-mail address: xiangming.xu@emr.ac.uk

* The $\boldsymbol{e}$-Xtra logo stands for "electronic extra" and indicates that Figure 2 appears in color online.

http://dx.doi.org/10.1094/PHYTO-10-12-0266-R

(C) 2013 The American Phytopathological Society agreement with most published experimental results (23). This meanfield model was then modified to incorporate the effects of fluctuating conditions on the BCA and pathogen development in order to assess whether combined use of a mycoparasitic BCA with a competitive one leads to synergy under temporally fluctuating conditions (25). The results suggested that combined use of a mycoparasitic BCA with a competitive one is unlikely to result in synergy.

Combined use of BCAs may be expected to result in synergy if two BCAs occupied different niches because, under this situation, the advantage of each individual BCA over the other within a given niche is more or less invariant over time. This is particularly the case when a pathogen is able to infect different host tissues, such as Botrytis cinerea (leaves, flowers, and fruit), but two BCAs may preferentially occupy different host tissues. The improved efficacy from combined use of two BCAs that occupy different niches was observed for $B$. cinerea on tomato $(14,15)$. Spatial heterogeneity in pathogen development and biocontrol is conceptually related to the concept of pathogen refuge size (13). Previous modeling also showed that incorporating spatial heterogeneity led to BCA persistence under conditions where homogeneous mixing predicted BCA extinction (7).

In this study, we modified the previous mean-field deterministic model of combined use of two BCAs (24) to study the effects of spatial heterogeneity on combined use of two competitive BCAs $(\mathrm{C} / \mathrm{C})$, or a mycoparasitic BCA with a competitive one $(\mathrm{C} / \mathrm{M})$, or two mycoparasitic BCAs (M/M). Specifically, the modified model considers two patches (habitats) to which the two BCAs are differentially adapted. There is an equal symmetric dispersal of BCAs 
and the pathogen between the two patches. We investigated the efficacy of combined use of two hypothetical BCAs in relation to (i) biocontrol activities of two BCAs in each patch, (ii) the relative size of the two patches, and (iii) dispersal between the two patches.

\section{THEORY AND APPROACHES}

Model description and numerical evaluation. Numerical simulations were used to study the effects of spatial heterogeneity on the biocontrol efficacy in relation to combined use of two hypothetical BCAs. The present model was based on the generic mean-field model developed by Jeger et al. (12), as modified for inundative application of one BCA or two BCAs $(24,26)$. In these models, host tissue was divided into several mutually exclusive categories (e.g., healthy susceptible, healthy resistant, infectious disease, post-infectious, and area occupied by BCA). Each biocontrol mechanism (competition, antibiosis, mycoparatism, and induced resistance) was then represented as an interaction or interactions between appropriate host tissue types, with the strength of the interactions represented by one or more model parameters. For example, a mycoparasitic BCA was assumed to have a better ability of colonizing (infecting) diseased tissues than healthy susceptible tissues. On the other hand, a competitive BCA was assumed to colonize only healthy susceptible tissues but not diseased tissues. Because combined use of a mycoparasitic and a competitive BCA is generally most effective and leads to complex damped-cyclic epidemics (24), the present study focused on the use of three two BCA combinations: $\mathrm{C} / \mathrm{C}, \mathrm{C} / \mathrm{M}$, and $\mathrm{M} / \mathrm{M}$. In addition, for these three combinations, the original model becomes simpler because the category of healthy resistant tissues (hence, the related four parameters) is no longer necessary.

We assumed that (i) there are two patches; (ii) within a single patch there is homogeneous mixing among the pathogen, BCAs and host; and (iii) two BCAs occupy the same type of host tissues as the pathogen. The patch scale is not fixed; it could be different trees within an orchard, or two different sections within a single field but experiencing significantly different microclimatic conditions, or even two different host tissue types (e.g., flowers and leaves). Within a single patch, the BCA-pathogen-host dynamics follow the same set of differential equations as under homogeneous conditions (24), except that the present study considered only competitive and mycoparasitic BCAs and assumed a constant level of pathogen and BCA dispersal between the two patches.

Host tissue was divided into the following mutually exclusive categories: healthy susceptible $\left(H_{S}\right)$, infectious disease $(I)$, removed disease (post-infectious) $(R)$, and area occupied by BCA $(B)$. The model assumes that hosts are divided into two patches with a ratio of host tissue $p:(1-p)[0 \leq p \leq 1]$ between the two patches, representing the relative size of the two patches. A subscript of 1 or 2 in $H_{S}, I$, and $R$ indicates the patch identity. Similarly, BCA-occupied host area $(B)$ is divided into four components: area occupied by each of two BCAs in the two patches. The first and second subscript in the $B$ state variable indicates the BCA and patch identity, respectively. There is a constant and symmetric dispersal $(g)$ of the pathogen and BCAs among the two patches. The full model is then
The 10 equations add to zero; that is, a constant host population size (the 10 state variables add to 1.0). Descriptions and default value for each model parameter are given in Table 1 . The rate of the $i$ th BCA colonizing healthy host tissue $\left(H_{S}\right)$ in the $j$ th patch $\left(c_{i j}\right)$ represents the competitive ability of a competitive BCA. Similarly, $m_{i j}$ refers to the rate of the $i$ th BCA colonizing diseased host tissue in the $j$ th patch, hence representing the biocontrol potential of a mycoparasitic BCA. A constant rate of mortality $(f)$ was assumed for both BCAs and dead BCA occupied-tissues returned to healthy susceptible tissue, which effectively simulates host growth. The pathogen infects healthy host tissues at a rate $b$ with a loss of infectiousness rate $h$, which does not vary between the two patches.

Simulation studies. Numerical studies were conducted to evaluate biocontrol efficacy of individual and combined use of BCAs in relation to a range of model parameters in the two patches. $\mathrm{Nu}-$ merical simulation (by the Modified Extended Backward Differentiation Equation Implicit method) was carried out in Maple 16. There are three possible combinations of two BCAs: a competitive BCA with another competitive one $(\mathrm{C} / \mathrm{C})$, a competitive $\mathrm{BCA}$ with a mycoparasitic one $(\mathrm{C} / \mathrm{M})$, and a mycoparasitic BCA with another mycoparasitic one (M/M).

Relative sizes of the two patches. The ratio of the two patch sizes is $p:(1-p)$; that is, $\left(H_{S 1}+I_{1}+R_{1}+B_{11}+B_{21}\right):\left(H_{S 2}+I_{2}+\right.$ $\left.R_{2}+B_{12}+B_{22}\right)=p:(1-p)$. Eleven $p$ values were studied from 0 to 1.0 , at an increment of 0.1 . For the $\mathrm{C} / \mathrm{C}$ and $\mathrm{M} / \mathrm{M}$ combinations, only values of $p$ of $0-0.5$ were considered because of the symmetry (e.g., $p=0.1$ is equivalent to $p=0.9$ ). Four values of pathogen or BCA dispersal $(g)$ among the two patches were studied: 0, 0.001, 0.01, and 0.1.

BCA activity in the two patches. For a competitive BCA, its differential development or biocontrol efficacy in the two patches was expressed as $c_{12}=q c_{11}$, where five $q$ values $(0,0.25,0.5$, 0.75 , and 1.0) were considered; thus, this BCA is better (or equally) adapted to patch 1 than to patch 2 . When $q=0$, this BCA cannot survive or develop in patch 2. For the second competitive $\mathrm{BCA}$, this was reversed; namely, $c_{21}=q c_{22}$. Similarly, when two mycoparasitic BCAs were used in combination, the relative biocontrol efficacy in the two patches was expressed as $m_{12}=$ $q m_{11}$ and $m_{21}=q m_{22}$ for the first and second BCA, respectively. For the $\mathrm{C} / \mathrm{M}$ combination, the parameter values in the two patches was expressed as $c_{12}=q c_{11}$ and $m_{21}=q m_{22}$ for the mycoparasitic and competitive BCA, respectively.

In total, 21 different $c_{i j}$ (or $m_{i j}$ ) values were evaluated for a BCA in its better adapted patch; $c_{i j}$ (or $\left.m_{i j}\right)$ was 0.2 to 0.6 at increments of 0.02 .

Initial condition and spray coverage. Within patch 1 (or 2), the pathogen was assumed to be present at the level of $0.001 p$ [or $0.001(1-p)]$ at the point entering into the infectious period. BCAs were introduced at the onset of an epidemic. Only a single spray coverage was considered because the previous study (26) showed that the effect of spray coverage of 50 to $90 \%$ on biocontrol efficacy was small. The coverage of a single BCA application was assumed to be $50 \%$ and, hence, the total coverage of combined use of two BCAs under the assumption of independent

$$
\begin{aligned}
& \mathrm{d} H_{S 1} / \mathrm{dt}=-H_{S 1}\left[b\left(I_{1}+g I_{2}\right)+c_{11}\left(B_{11}+g B_{12}\right)+c_{21}\left(B_{21}+g B_{22}\right)\right]+f\left(B_{11}+B_{21}\right) \\
& \mathrm{d} B_{11} / \mathrm{dt}=c_{11}\left(B_{11}+g B_{12}\right) H_{S 1}+m_{11}\left(I_{1}+R_{1}\right)\left(B_{11}+g B_{12}\right)-f B_{11} \\
& \mathrm{~d} B_{21} / \mathrm{dt}=c_{21}\left(B_{21}+g B_{22}\right) H_{S 1}+m_{21}\left(I_{1}+R_{1}\right)\left(B_{21}+g B_{22}\right)-f B_{21} \\
& \mathrm{~d} I_{1} / \mathrm{dt}=b H_{S 1}\left(I_{1}+g I_{2}\right)-I_{1}\left[m_{11}\left(B_{11}+g B_{12}\right)+m_{21}\left(B_{21}+g B_{22}\right)+h\right] \\
& \mathrm{d} R_{1} / \mathrm{dt}=h I_{1}-R_{1}\left[m_{11}\left(B_{11}+g B_{12}\right)+m_{21}\left(B_{21}+g B_{22}\right)\right] \\
& \mathrm{d} H_{S 2} / \mathrm{dt}=-H_{S 2}\left[b\left(I_{2}+g I_{1}\right)+c_{12}\left(B_{12}+g B_{11}\right)+c_{22}\left(B_{22}+g B_{21}\right)\right]+f\left(B_{12}+B_{22}\right) \\
& \mathrm{d} B_{12} / \mathrm{dt}=c_{12}\left(B_{12}+g B_{11}\right) H_{S 2}+m_{12}\left(I_{2}+R_{2}\right)\left(B_{12}+g B_{11}\right)-f B_{12} \\
& \mathrm{~d} B_{22} / \mathrm{dt}=c_{22}\left(B_{22}+g B_{21}\right) H_{S 2}+m_{22}\left(I_{2}+R_{2}\right)\left(B_{22}+g B_{21}\right)-f B_{22} \\
& \mathrm{~d} I_{2} / \mathrm{dt}=b H_{S 2}\left(I_{2}+g I_{1}\right)-I_{2}\left[m_{12}\left(B_{12}+g B_{11}\right)+m_{22}\left(B_{22}+g B_{21}\right)+h\right] \\
& \mathrm{d} R_{2} / \mathrm{dt}=h I_{2}-R_{2}\left[m_{12}\left(B_{12}+g B_{11}\right)+m_{22}\left(B_{22}+g B_{21}\right)\right]
\end{aligned}
$$


with overlapping coverage was estimated to be $75 \%[=50 \%+$ $(100 \%-50 \%) \times 50 \%$ ], equally divided between the two BCAs. However, if one of the two BCAs cannot survive or develop in one patch (i.e., $q=0$ ), the spray coverage is assumed to be $50 \%$ for the other BCA (instead of 37.5\%). Thus, for the application with a single BCA or two BCAs with $q=0,0.5 \times 0.999 p$ [or $0.5 \times 0.999(1-p)$ ] was allocated to the relevant $\mathrm{BCA}$ in patch 1 (or 2). Similarly, for combined use of two BCAs with $q>$ $0,0.375 \times 0.999 p$ [or $0.375 \times 0.999(1-p)$ ] was allocated to each $\mathrm{BCA}$ in patch 1 (or 2). The remaining host tissue was allocated to the $H_{S}$.

Simulation runs. Thus, in total, there were 4,620 parameter combinations ( 21 biocontrol efficacy parameters, five differentials in the biocontrol efficacy $[q]$ among the two patches, 11 relative sizes $[p]$ of the two patches, and four dispersal values $[g]$ between the two patches) for the C/M combination. For the $\mathrm{C} / \mathrm{C}$ and $\mathrm{M} / \mathrm{M}$ combinations, there were 2,520 parameter combinations each because there were only six values for $p$. Similarly, individual use of component BCAs was also evaluated under the same parameter combinations. In addition, for each combination of patch size and dispersal, a pathogen-only model was run as the basis for comparison (to calculate control efficacy).

Data analysis. For each simulated disease dynamic, the total disease over the two patches was obtained at each time step, and area under the disease progress curve (AUDPC) was then calculated for the total disease. Biocontrol efficacy was then estimated as $E_{b}=\left[100\left(A U D P C_{P}-A U D P C_{B}\right)\right] / A U D P C_{P}$, where $A U D P C_{P}$ and $A U D P C_{B}$ are the AUDPC values for the simulated epidemics without and with BCAs, respectively, under the same combination of patch size and dispersal parameter values. The expected biocontrol efficacy for combined use of two BCAs was estimated based on the Bliss independence with overlapping (1); that is, $E_{M}=E_{1}+E_{2}-E_{1} E_{2}$, where $E_{1}$ and $E_{2}$ are the efficacies achieved when the two individual BCAs were applied alone under the same conditions.

Four-way analysis of variance was used to assess the overall effect of simulation factors on the biocontrol efficacy resulting from combined use of each of the three two-BCA combinations. The four factors were BCA activity $(r)$, patch differences in BCA activity $(q)$, relative patch size $(p)$, and dispersal between two patches $(g)$. In addition, the efficacy from combined use was also expressed as a ratio with the expected efficacy (based on the Bliss independence) or the efficacy of the more efficacious BCA used alone. Logit-transformation-that is, $\ln \left[E_{b} /\left(1-E_{b}\right)\right]$ - was applied to the biocontrol efficacy whereas no transformation was necessary for the two relative efficacy measures.

\section{RESULTS}

Overall efficacy. Biocontrol efficacy resulting from combined use of two BCAs varied greatly with model parameter values, covering the whole range (0 to $100 \%$ ). Nearly 50 and $75 \%$ of simulation runs had an efficacy $<20$ and $40 \%$, respectively. Averaged over all the simulation variables, combined use of two myco- parasitic BCAs (M/M) led to the least efficacy (21.5\%), compared with $29.6 \%(\mathrm{C} / \mathrm{C})$ and $29.0 \%(\mathrm{M} / \mathrm{M})$.

As expected, biocontrol efficacy was strongly affected by the BCA activity level ( $r$ ); increasing $r$ led to increased efficacy (Fig. 1). However, the degree of this increase in the efficacy with the increasing $r$ became gradually greater for the $\mathrm{C} / \mathrm{C}$ and $\mathrm{C} / \mathrm{M}$ combinations than for the $\mathrm{M} / \mathrm{M}$ combination when $r$ exceeded 0.44 (Fig. 1). For the $\mathrm{C} / \mathrm{C}$ and $\mathrm{C} / \mathrm{M}$ combinations, $>93$ and $73 \%$ of total variation in the logit-transformed efficacy was attributed to the changes in $r$ (i.e., $c_{i j}$ or $m_{i j}$ ), respectively (Table 2), compared with $48 \%$ for the $\mathrm{M} / \mathrm{M}$ combination.

For the $\mathrm{C} / \mathrm{M}$ combination, relative patch size $(p)$ and its interaction with the BCA activity $(r)$ also accounted for nearly 13 and $7 \%$ of the total variation, respectively. For the M/M combination, even greater proportion of the variability was attributable to $p(33 \%)$ and its interaction with $r(18 \%)$ (Table 2). Increasing the size difference between the two patches (i.e., increasing difference between $p$ and 0.5 ) led to increasing biocontrol efficacy for a given set of parameter values. Therefore, the average biocontrol efficacy was the lowest when the two patches were of the same size (i.e., $p=0.5$ ). The effect of the size difference between the two patches on biocontrol efficacy was also affected by the biocontrol mechanisms involved. The effect was least and greatest for the $\mathrm{C} / \mathrm{C}$ and $\mathrm{M} / \mathrm{M}$ combinations, respectively. For the $\mathrm{C} / \mathrm{C}$ and $\mathrm{M} / \mathrm{M}$ combinations, increasing $p$ from 0 to 0.5 led to the reduction of overall efficacy from 36.2 to $21.8 \%$ and from 40.0 to $7.7 \%$, respectively. For the $\mathrm{C} / \mathrm{M}$ combination, increasing $p$ from 0 to 0.5

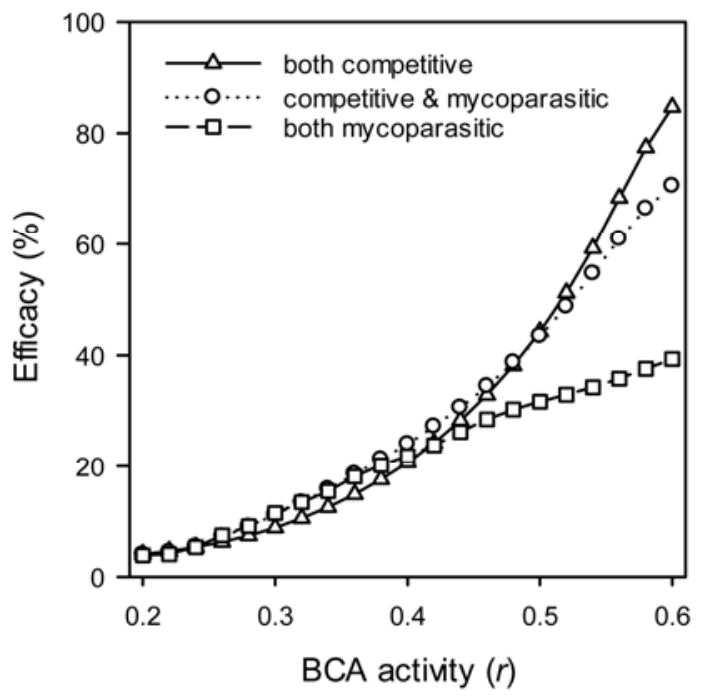

Fig. 1. Biocontrol efficacy resulting from combined use of two biocontrol agents (BCAs) at 21 different values of the model parameter describing the biocontrol activity $(r)$ in one of the two patches to which one of two BCAs is better adapted. Each point is an average of 120 numerical evaluations (i.e., combinations of other parameter values) of the model for the competitive/ competitive and mycoparasitic/mycoparasitic combinations, and 220 evaluations for the competitive and mycoparasitic combination.

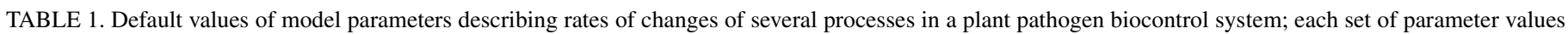
represents a biocontrol agent (BCA) with a single main biocontrol mechanism as specified ${ }^{\mathrm{a}}$

\begin{tabular}{|c|c|c|c|c|}
\hline Parameter $\left(\right.$ time $\left.^{-1}\right)$ & Description & Mycoparasitism & Competition & Pathogen \\
\hline$c_{11}, c_{12}$ & BCA1 colonizing healthy tissue in patch 1 and 2 , respectively & 0.01 & See text & $\ldots$ \\
\hline$c_{21}, c_{22}$ & BCA2 colonizing healthy tissue in patch 1 and 2 , respectively & 0.01 & See text & $\ldots$ \\
\hline$m_{11}, m_{12}$ & BCA1 colonizing diseased tissue in patch 1 and 2 , respectively & See text & 0.01 & $\ldots$ \\
\hline$m_{21}, m_{22}$ & BCA2 colonizing diseased tissue in patch 1 and 2 , respectively & See text & 0.01 & $\ldots$ \\
\hline$f$ & BCA mortality (to susceptible) & 0.2 & 0.2 & $\ldots$ \\
\hline$g$ & Dispersal for either pathogen or BCAs between two patches & $\ldots$ & $\ldots$ & See text \\
\hline$b$ & Pathogen infection rate $\left(\right.$ time $\left.^{-1}\right)$ & $\ldots$ & $\ldots$ & 0.2 \\
\hline$H$ & Pathogen infectious rate $\left(\right.$ time $\left.^{-1}\right)$ & $\ldots$ & $\ldots$ & 0.05 \\
\hline
\end{tabular}

a Two key BCAs ( $c$ for the competitive BCA and $m$ for the mycoparasitic BCA) parameters vary between two patches for each BCA. 
led to the reduction of overall efficacy from 41.2 to $17.1 \%$, and further increasing $p$ from 0.5 to 1.0 then led to the efficacy increase from 17.1 to $40.0 \%$. Interactions between $p$ and $r$ are shown in Figure 2 for three combinations, illustrating the largest interaction for the $\mathrm{M} / \mathrm{M}$ combination (i.e., the change in efficacy with the change in $p$ varied considerably with $r$ ).

Comparison of the efficacy from combined use of two BCAs with the more efficacious BCA. In the majority of the numerical simulations $(6,966$ of 9,660), combined use of two BCAs led to greater efficacy than the more efficacious BCA used alone (Fig. 3A). In $\approx 59,80$, and $70 \%$ of simulated epidemics, combined use of two BCAs led to greater efficacy than the more efficacious $\mathrm{BCA}$ for the $\mathrm{C} / \mathrm{C}, \mathrm{C} / \mathrm{M}$, and $\mathrm{M} / \mathrm{M}$ combinations, respectively.

The ratio of the efficacy from combined use with that of the more efficacious BCA was greatly affected by the relative patch size $(p)$, accounting for 61,44 , and $62 \%$ of the total variation for the $\mathrm{C} / \mathrm{C}, \mathrm{C} / \mathrm{M}$, and $\mathrm{M} / \mathrm{M}$ combinations, respectively (Table 2).
When the difference in the size between the two patches became smaller (i.e., $p \rightarrow 0.5$ ), the efficacy ratio increased sharply (Fig. 4A). In addition, the contribution by the interaction of $p$ with the difference in BCA activity between two patches $(q)$ was also large (Table 2), particularly for the $\mathrm{C} / \mathrm{C}$ combination (Fig. 5). Combined use more likely led to greater ratios when there was less difference in patch sizes (i.e., $p \rightarrow 0.5$ ) or $q \rightarrow 1.0$ (Fig. 5).

For the $\mathrm{C} / \mathrm{M}$ and $\mathrm{M} / \mathrm{M}$ combinations, the BCA activity rate $(r)$ and its interaction with $p$ also accounted for a considerable proportion of the total variation in the efficacy ratio (Table 2). In both combinations, increasing $r$ generally led to less difference between smaller efficacy ratios (Fig. 4B). For the C/M, both the high and low $r$ values as well as $p \rightarrow 0.5$ (less difference in patch sizes) led to greater differences in the efficacy between the more efficacious BCA and the combined use (Fig. 6B) whereas, for the $\mathrm{C} / \mathrm{C}$ and $\mathrm{M} / \mathrm{M}$ combinations, lower $r$ values and $p \rightarrow 0.5$ resulted in improved efficacy from combined use over the more efficacious BCA used alone (Fig. 6A and C).

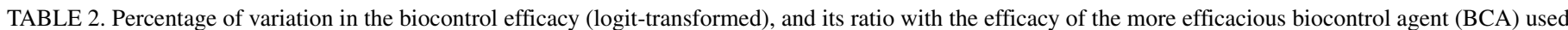
alone or with the expected efficacy based on the Bliss independence attributable to simulation variables (model parameters) and their interactions

\begin{tabular}{|c|c|c|c|c|c|c|c|c|c|}
\hline \multirow[b]{3}{*}{ Source ${ }^{a}$} & \multicolumn{9}{|c|}{ Variation $(\%)^{\mathrm{b}}$} \\
\hline & \multicolumn{3}{|c|}{ Both competitive } & \multicolumn{3}{|c|}{ Competitive with mycoparasitic } & \multicolumn{3}{|c|}{ Both mycoparasitic } \\
\hline & Efficacy & Better & Expected & Efficacy & Better & Expected & Efficacy & Better & Expected \\
\hline$d$ & 0.05 & 0.01 & 0.05 & 0.04 & 0.24 & 0.03 & 0.15 & 0.12 & 0.03 \\
\hline$r$ & 93.26 & 1.31 & 25.51 & 73.63 & 6.49 & 24.48 & 47.96 & 6.61 & 1.53 \\
\hline$q$ & 0.33 & 4.69 & 59.50 & 2.94 & 1.67 & 31.73 & 0.40 & 2.09 & 67.20 \\
\hline$p$ & 4.90 & 60.55 & 0.45 & 12.72 & 44.23 & 3.97 & 33.44 & 61.56 & 1.07 \\
\hline d.r & 0.04 & 0.19 & 0.06 & 0.01 & 0.07 & 0.02 & 0.00 & 0.04 & 0.02 \\
\hline$d . q$ & 0.03 & 0.17 & 0.28 & 0.03 & 0.24 & 0.04 & 0.00 & 0.07 & 0.19 \\
\hline$q . r$ & 0.03 & 2.48 & 5.19 & 1.56 & 1.54 & 9.37 & 0.07 & 1.74 & 4.95 \\
\hline d.p & 0.04 & 0.64 & 0.21 & 0.03 & 2.10 & 0.12 & 0.07 & 1.46 & 0.21 \\
\hline$r . p$ & 1.20 & 3.56 & 2.22 & 6.88 & 15.46 & 5.28 & 17.57 & 8.95 & 4.17 \\
\hline$q . p$ & 0.03 & 21.82 & 1.85 & 0.76 & 12.29 & 11.33 & 0.19 & 13.76 & 10.17 \\
\hline d.r.q & 0.02 & 0.25 & 0.15 & 0.02 & 0.08 & 0.01 & 0.00 & 0.05 & 0.01 \\
\hline d.r.p & 0.03 & 0.44 & 0.18 & 0.02 & 0.97 & 0.12 & 0.07 & 0.19 & 0.11 \\
\hline d.q.p & 0.03 & 1.18 & 0.65 & 0.02 & 2.22 & 0.19 & 0.00 & 1.53 & 0.57 \\
\hline r.q.p & 0.02 & 2.06 & 3.31 & 1.29 & 11.47 & 13.25 & 0.10 & 1.78 & 9.62 \\
\hline Residual & 0.02 & 0.64 & 0.37 & 0.02 & 0.94 & 0.07 & 0.00 & 0.09 & 0.14 \\
\hline
\end{tabular}

a Source of variation: dispersal $(d)$, BCA activity $(r)$, BCA activity difference in two patches $(q)$, and patch size $(p)$.

${ }^{\mathrm{b}}$ Better $=$ ratio with better $\mathrm{BCA}$ and Expected $=$ ratio with the expected.
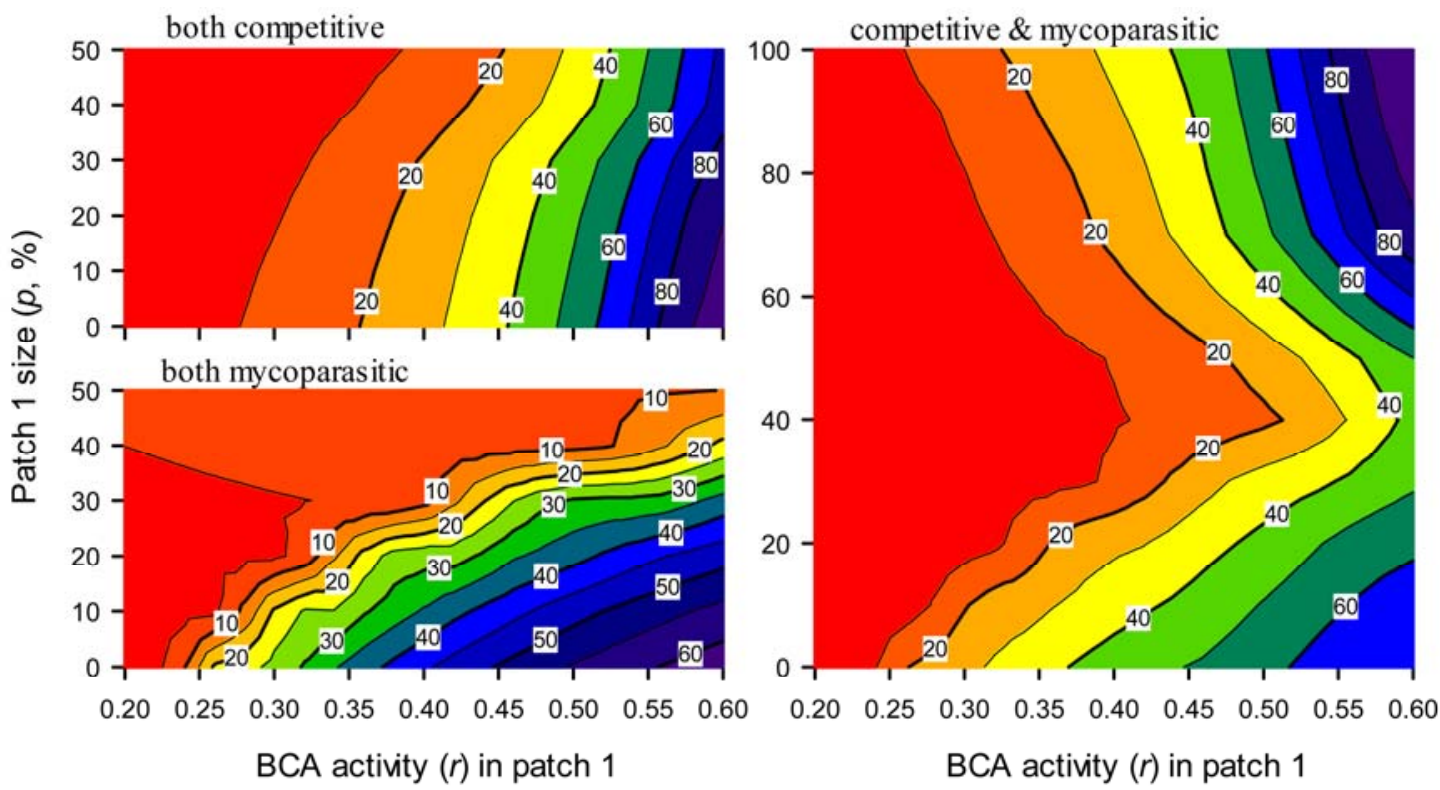

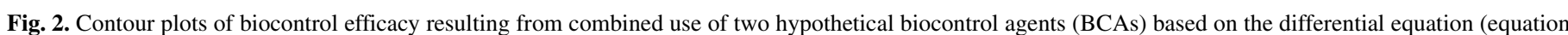

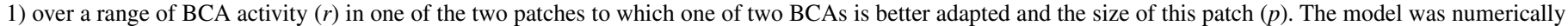

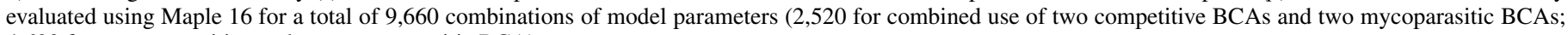
4,620 for one competitive and one mycoparasitic BCA). 
Comparison of the efficacy from combined use of two BCAs with the expectation based on Bliss independence. There was a considerable number of simulated epidemics $(2,046$ of 9,660 simulations) where combined use of two BCAs resulted in synergy (Fig. 3B). Overall, the C/M combination led to the greatest efficacy ratio (0.88) between the simulated and the expected on the basis of the Bliss independence for the combined use, whereas the $\mathrm{C} / \mathrm{C}$ and $\mathrm{M} / \mathrm{M}$ combinations had similar efficacy ratios (0.83).

The difference in the BCA activity between the two patches $(q)$ accounted for the most variation in the efficacy ratio, particularly for the $\mathrm{C} / \mathrm{C}(60 \%)$ and $\mathrm{M} / \mathrm{M}(67 \%)$ combinations (Table 2). The efficacy ratio decreased with increasing $q$ (i.e., decreasing difference in biocontrol activity between the two patches) (Fig. 4C). There were nearly 26,19 , and $15 \%$ of simulations with the efficacy ratio $>1.0$ for the $\mathrm{C} / \mathrm{M}, \mathrm{C} / \mathrm{C}$, and $\mathrm{M} / \mathrm{M}$ combinations, respectively. For both the $\mathrm{C} / \mathrm{C}$ and $\mathrm{M} / \mathrm{M}$ combinations, nearly all simulations with the ratio $>1.0$ were for cases where a single $\mathrm{BCA}$ can survive in one patch only, which is in contrast to the situation for the $\mathrm{C} / \mathrm{M}$ combination (Fig. 7). For the $\mathrm{C} / \mathrm{M}$ and $\mathrm{C} / \mathrm{C}$ combinations, the effect of BCA activity $(r)$ was also large (Table 2 ), accounting for approximately a quarter of the total variation; the ratio increased with increasing $r$ (Fig. 4B). For the C/M and
$\mathrm{M} / \mathrm{M}$ combinations, the ratio was also considerably affected by the interaction between relative patch size $(p)$ and $q$ (Fig. 8). For all three combinations, the interaction between $r$ and $q$ was also noticeable, accounting 5 to $10 \%$ of the total variability.

\section{DISCUSSION}

Previous theoretical modeling studies have shown that combined use of two hypothetical BCAs is unlikely to result in synergy under homogeneous (24) or temporally fluctuating conditions (25). The present modeling study investigated combined use of two hypothetical BCAs under spatially heterogeneous conditions for BCAs involving two specific mechanisms (competition and mycoparasitism). The findings are different from those of previous modeling results, in that combined use of two BCAs would lead, in most cases, to control efficacies that are greater than the more efficacious BCA used alone and, in some cases, to synergy based on the Bliss independence criterion.

Combined use of two BCAs is more likely to result in efficacies greater than the more efficacious BCA used alone under conditions where two patches have similar sizes. When one patch is too large relative to the other, the efficacy resulting from com-
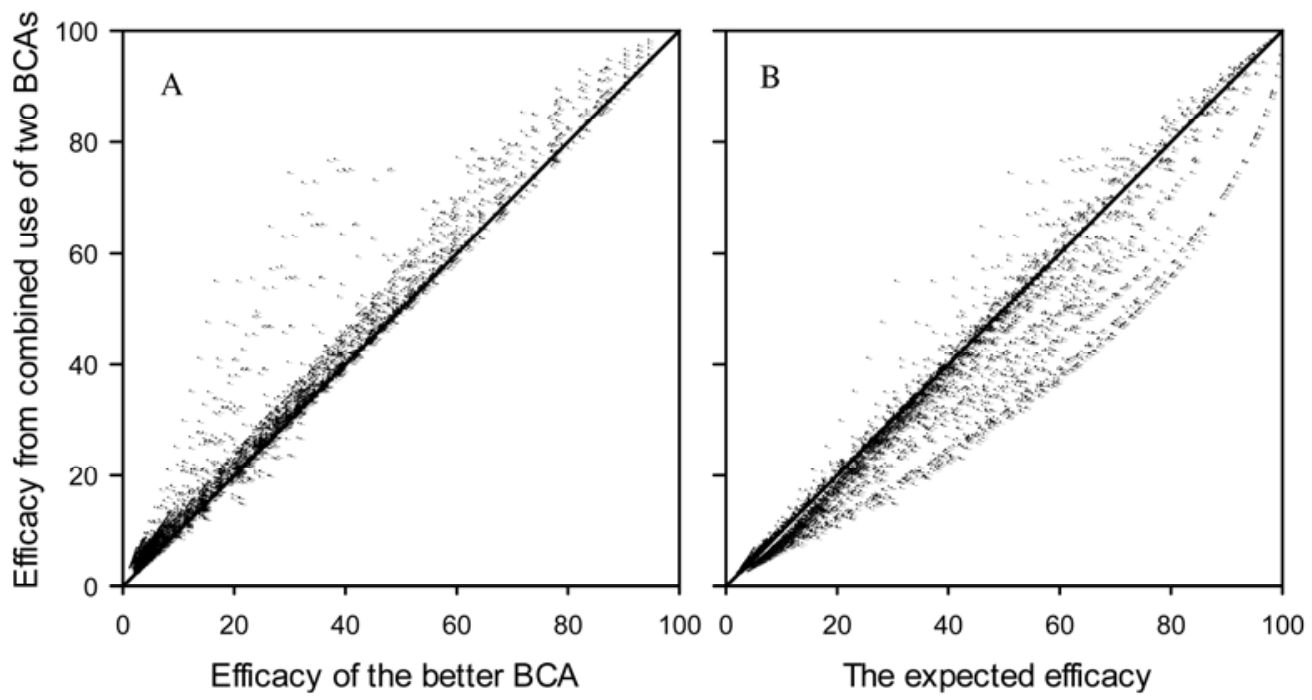

Fig. 3. Plot of simulated biocontrol efficacy resulting from combined use of two hypothetical biocontrol agents (BCAs) based on the differential equation (equation 1) against $\mathbf{A}$, the efficacy of the more efficacious hypothetical BCA used alone or $\mathbf{B}$, the efficacy expected on the basis of the Bliss independence. Solid lines are the 1:1 lines (i.e., when the efficacies are equal). The model was numerically evaluated using Maple 16 for a total of 9,660 combinations of model parameter values.
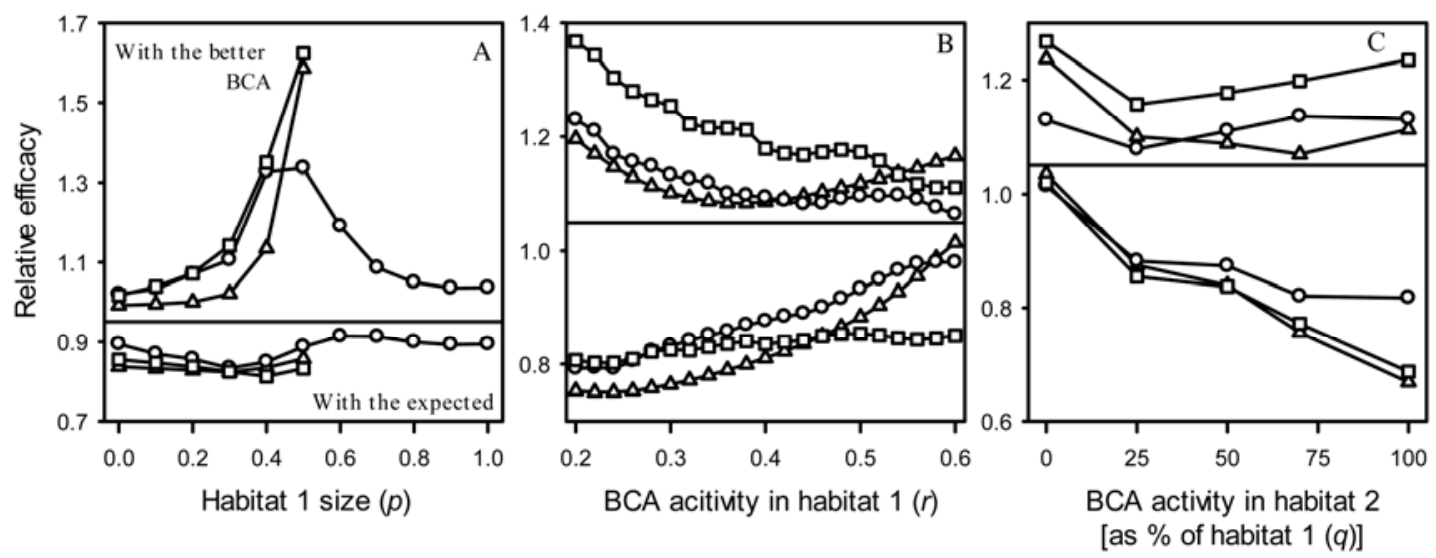

Fig. 4. Ratio of efficacies from combined use of two biocontrol agents (BCAs) with the more efficacious BCA used alone (top frame graph) or the efficacy expected on the basis of Bliss independence (bottom frame graph) in relation to $\mathbf{A}$, size of patch $1(p)$; $\mathbf{B}$, activity of one of the two BCAs $(r)$ in patch 1; and $\mathbf{C}$, the difference in biocontrol activity between the two patches $(q)$ for a given BCA. Triangle, circle, and square symbols represent the combined use of two competitive, one competitive with one mycoparasitic, and two mycoparasitic BCAs, respectively. 
bined use is largely attributable to the single BCA that is better adapted to the larger patch. Consequently, the difference in the efficacy between the combined use and the more efficacious one is likely to be small. Even when two BCAs are adapted equally

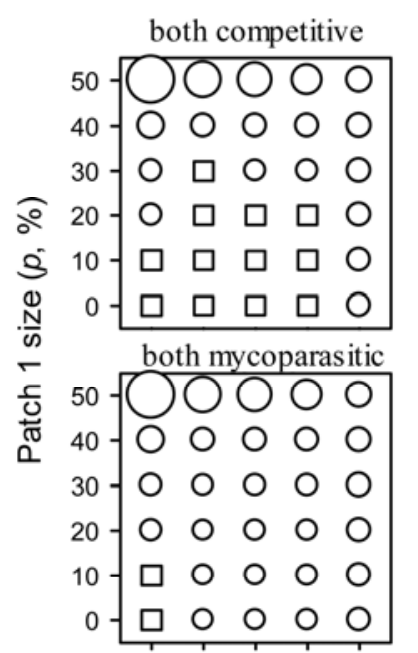

$0 \quad 25 \quad 50 \quad 75 \quad 100$ competitive

\& mycoparasitic

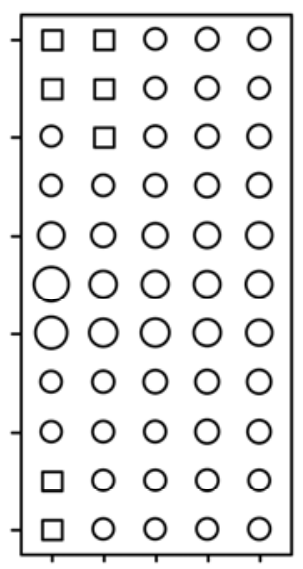

$\begin{array}{lllll}0 & 25 & 50 & 75 & 100\end{array}$
BCA activity in patch 2 as $\%$ of patch $1(q)$

Fig. 5. Bubble plot of the average ratio of biocontrol efficacies from combined use of two hypothetical biocontrol agents (BCAs) with that of the more efficacious one used alone over each combination of patch size $(p)$ and difference in BCA activity between the two patches $(q)$. Square and circle symbols indicate that the ratio is $\leq 1.0$ or $>1.0$, respectively; symbol sizes represent their relative magnitude of the ratio. well to the two patches, their combined use, in most cases, can result in better control than the more efficacious BCA used alone. This differs from simulations under homogeneous or fluctuating conditions where the combined use generally results in efficacies similar to that of the more efficacious one $(24,25)$. Thus, spatial patchiness alone (without differential adaptation of BCAs or pathogens [i.e., $q=1.0$ in the present study]) could lead to differential biocontrol outcomes; the dispersal between the two patches of 0 to 0.1 did not sufficiently negate the effect of spatial patchiness on biocontrol outcomes. The relatively better performance of combined use of BCAs over the individual component BCAs used alone in a patchy environment is likely due to the reduced direct or indirect competition between BCAs under the patchy condition, compared with the homogeneous condition. Under the condition of two equal-size patches, for example, with no dispersal, the direct or indirect interaction between two BCAs is expected to be half of that experienced under homogeneous conditions. The importance of spatial patchiness (hence, dispersal gradient) on disease development is well known; for example, in the spread of invasive species in landscapes (16) and disease development in mixtures of cultivars (17). However, the effect of patchiness on biocontrol of plant diseases is less well understood. Several experimental studies suggested the importance of patch sizes or patchiness on biocontrol of insect pests $(10,21,22)$, depending on the complex interaction among the extent of spatial heterogeneity, prey or predator mobility and life cycles, and predator searching efficiency.

Moreover, under spatially heterogeneous conditions, combined use of two BCAs may result in synergistic interactions where the efficacy is greater than the expectation based on the Bliss independence. Synergy is more likely to result from situations where there is large differential adaptation of two BCAs in the two

\section{A: both competitive}

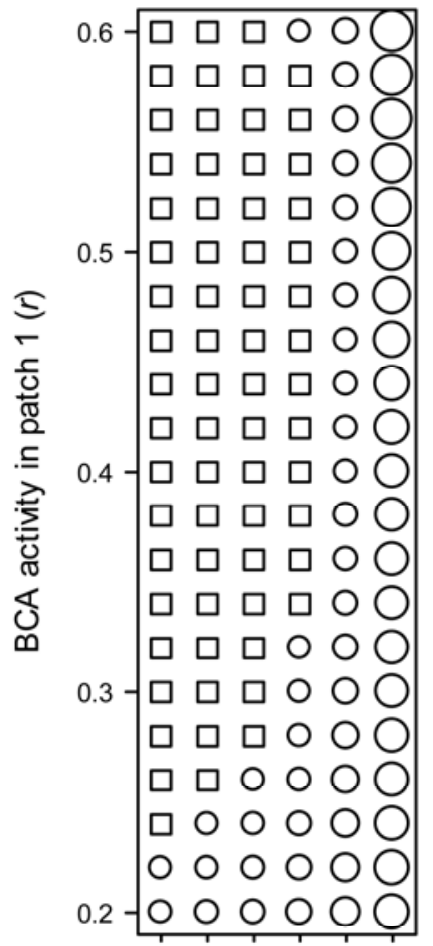

$\begin{array}{llllll}0 & 10 & 20 & 30 & 40 & 50\end{array}$
B: competitive \& mycoparasitic

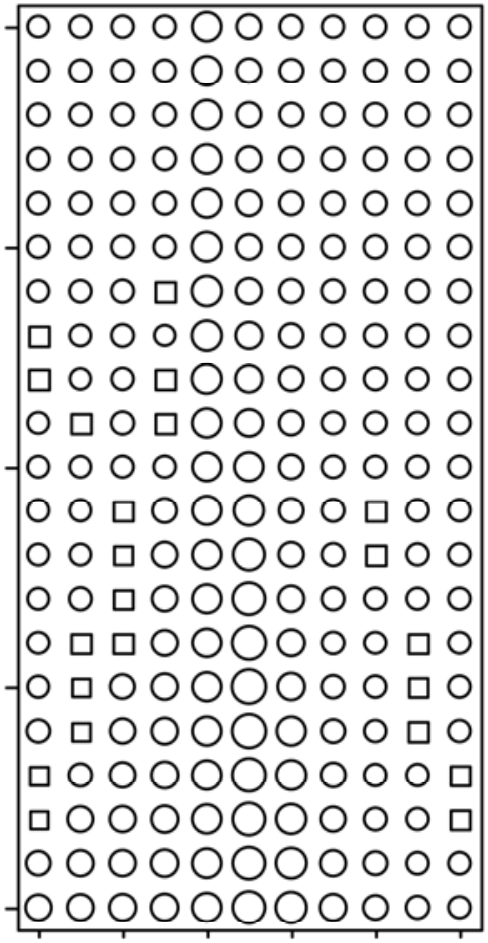

0
20
40

\section{C: both mycoparasitic}

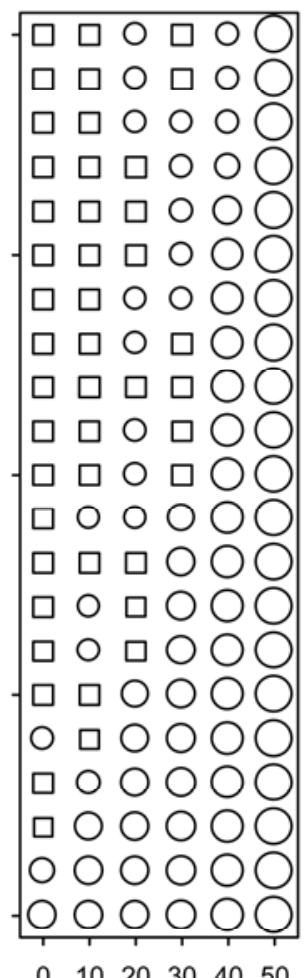

Patch 1 size $(p, \%)$

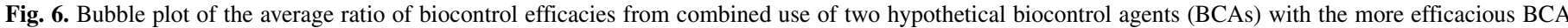

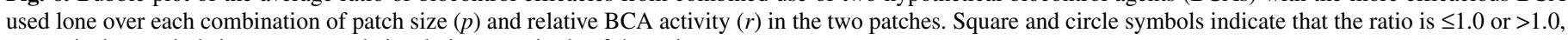
respectively; symbol sizes represent their relative magnitude of the ratio. 
patches. In extreme cases, one BCA survives in one patch only and the other survives in the other patch (i.e., no antagonism between the two BCAs is possible). Synergy under this condition can be understood intuitively in terms of a reduced pathogen refuge size (13). A special case of the extreme spatial patchiness in biocontrol is that when a pathogen is able to infect different host tissues but two BCAs may preferentially occupy different host tissues. The improved efficacy from combined use of two BCAs that occupy different niches was observed for $B$. cinerea on tomato $(14,15)$. The likelihood of synergy from combined use of two BCAs depends upon whether the two BCAs have similar biocontrol mechanisms. When the mechanism in the two BCAs is the same, combined use would less likely result in synergy, with most of synergistic cases restricted to situations where each BCA can only survive in one of the two patches. This is to be expected because there is a direct competition between the two BCAs for diseased tissues in the case of two mycoparasitic BCAs or for healthy tissues in the case of two competitive BCAs.

The extent of synergy (or lack of synergy) is primarily determined by the extent of differential adaptation of the two BCAs to the two patches. Increasing differential adaptation led to better performance of combined use relative to the expectation on the basis of Bliss independence. In addition, the relative performance was also greatly affected by the magnitude of BCA activity for the combinations involving at least one competitive BCA: the relative efficacy increased with the increasing BCA activity in the whole range studied. For competitive BCAs, BCA activity is important in determining its ability in colonizing host tissues relative to the pathogen; thus, increasing BCA activity is expected to lead to increasing efficacy. On the other hand, epidemic development in the presence of a mycoparasitic BCA is expected to follow a damped cyclic pattern $(12,26)$. Even at high parasitic rates, the disease level has to reach a certain level before parasitism by BCAs can reduce disease to a lower level; hence, a certain level of disease development is inevitable.

The present modeling study suggested that, under spatially heterogeneous conditions, combined use of two hypothetical BCAs may result in better disease control than the more efficacious BCA used alone and, indeed, may lead to synergy. However, this synergy has rarely been observed in experimental studies (23); most combinational treatments resulted in control efficacies less than (or similar to) the more efficacious one used alone and hence much less than the expected on the basis of the Bliss independence. This difference probably results from the fact that most controlled experiments were conducted under more-orless spatially homogeneous conditions. For example, BCAs were applied to wounded apple fruit and stored under controlled conditions $(2,3,11)$, detached leaves and incubated under in vitro condi- tions $(5,8)$, or seed $(19,20,27)$. Under these conditions, spatial heterogeneity is minimized, thus minimizing the likelihood of synergy between BCAs. Therefore, there appears to be a paradox about previous experimental research studies on combined use of BCAs. Researchers tried to demonstrate the existence of synergistic interactions among BCAs but conducted experiments under conditions that were not likely to result in synergy. Further experimental studies are needed to demonstrate whether synergistic interactions among BCAs occur under spatially heterogeneous conditions, hence providing a test of the model predictions. Such experiments may explore biocontrol on different tissue organs (e.g., flowers and fruit) or under different conditions (e.g., mixed plantations in open field and under protection).

In conclusion, the present modeling results suggest that synergy may result from combined use of BCAs under spatially heterogeneous conditions. The actual biocontrol efficacy achieved by combined use of two BCAs is generally higher when at least one component $\mathrm{BCA}$ has competition as its main biocontrol mechanism, especially when the biocontrol activity level is not very low.

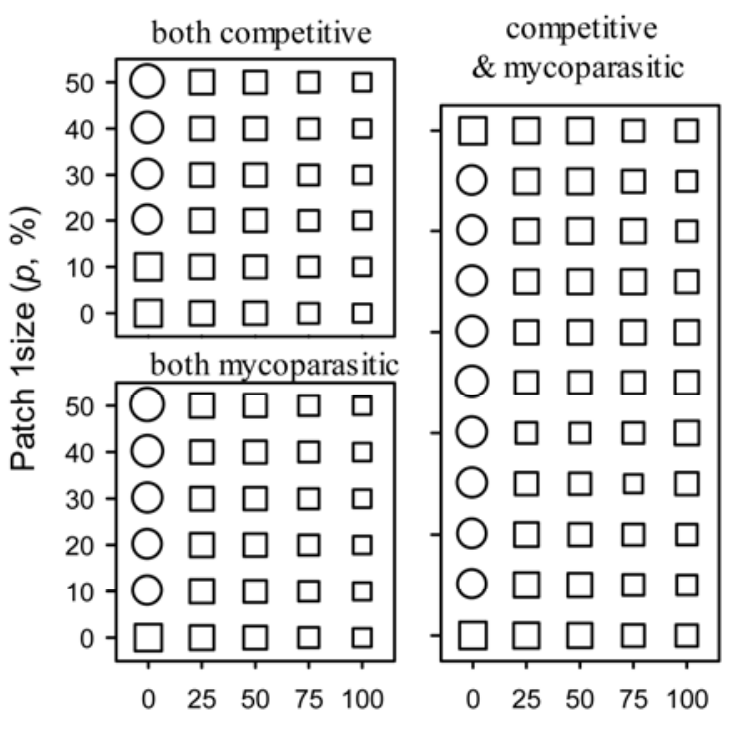

BCA activity in patch 2 as \% of patch $1(q)$

Fig. 8. Bubble plot of the average ratio of biocontrol efficacies from combined use of two hypothetical biocontrol agents (BCAs) with the efficacy expected on the basis of Bliss independence over each combination of patch size $(p)$ and difference in the BCA activity between the two patches $(q)$. Square and circle symbols indicate that the ratio is $\leq 1.0$ or $>1.0$, respectively; symbol sizes represent their relative magnitude of the ratio.
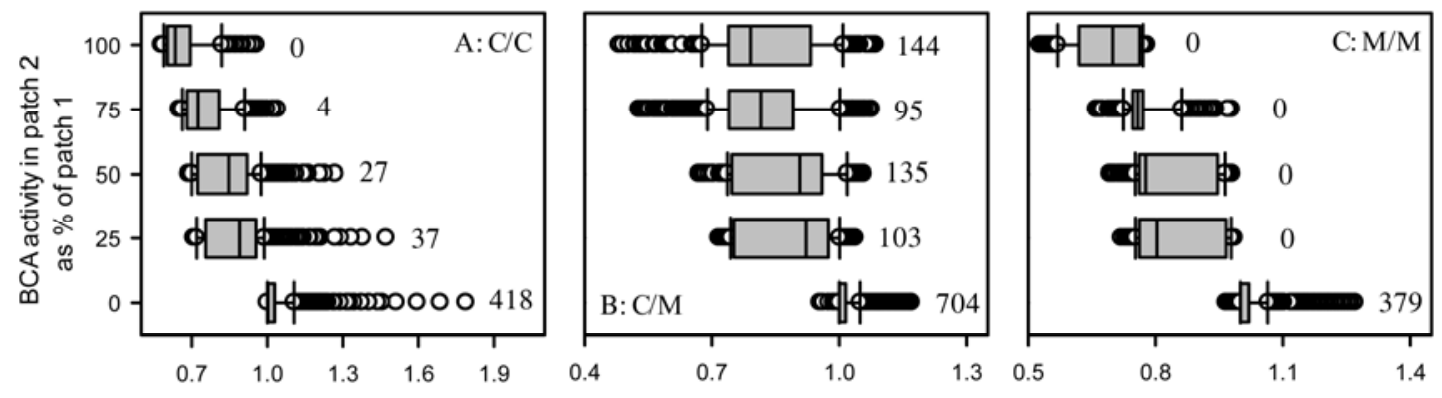

Ratio of simulated efficacy from combined use of two BCAs with the expected based on the Bliss independence

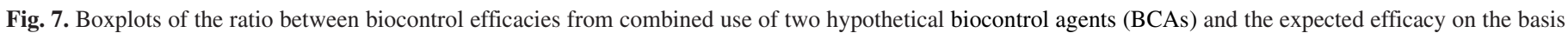

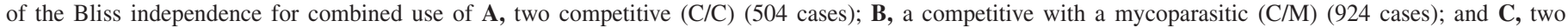

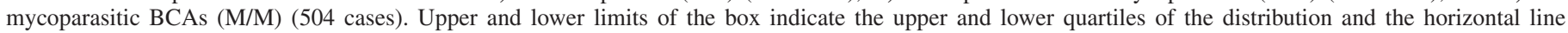

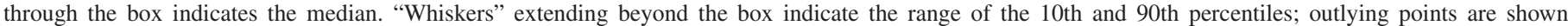

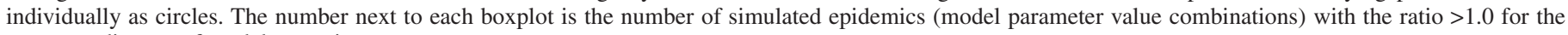
corresponding set of model scenarios. 
To be able to predict the performance of combined use of two BCAs, we need to understand not only the main biocontrol mechanisms involved but also the extent of patchiness and the level of biocontrol activity of individual BCAs in relation to external conditions.

\section{ACKNOWLEDGMENTS}

This research was funded by the UK Pesticide Safety Directorate (PSD), Department of Environment Food and Rural Affairs (Defra) (project number PS2119). X.-M. Xu is also partially supported by the 111 Project from Education Ministry of China (B07049).

\section{LITERATURE CITED}

1. Bliss, C. I. 1956. The calculation of microbial assays. Bacteriol. Rev. 20:243-258.

2. Calvo, J., Calvente, V., De Orellano, M. E., Benuzzi, D., and De Tosetti, M. I. S. 2003. Improvement in the biocontrol of postharvest diseases of apples with the use of yeast mixtures. Biocontrol 48:579-593.

3. Conway, W. S., Leverentz, B., Janisiewicz, W. J., Saftner, R. A., and Camp, M. J. 2005. Improving biocontrol using antagonist mixtures with heat and/or sodium bicarbonate to control postharvest decay of apple fruit. Postharvest Biol. Technol. 36:235-244.

4. Cunniffe, N., and Gilligan, C. 2011. A theoretical framework for biological control of soil-borne plant pathogens: Identifying effective strategies. J. Theor. Biol. 278:32-43.

5. Elliott, M., Shamoun, S. F., Sumampong, G., James, D., Masri, S., and Varga, A. 2009. Evaluation of several commercial biocontrol products on European and North American populations of Phytophthora ramorum. Biocontrol Sci. Technol. 19:1007-1021.

6. Freeman, S., Minz, D., Kolesnik, I., Barbul, O., Zveibil, A., Maymon, M., Nitzani, Y., Kirshner, B., Rav-David, D., Bilu, A., Dag, A., Shafir, S., and Elad, Y. 2004. Trichoderma biocontrol of Colletotrichum acutatum and Botrytis cinerea and survival in strawberry. Eur. J. Plant Pathol. 110:361370.

7. Gubbins, S., and Gilligan, C. A. 1997. Biological control in a disturbed environment. Philos. Trans. R. Soc. Lond. Ser. B Biol. Sci. 352:1935-1949.

8. Guetsky, R., Elad, Y., Shtienberg, D., and Dinoor, A. 2002. Improved biocontrol of Botrytis cinerea on detached strawberry leaves by adding nutritional supplements to a mixture of Pichia guilermondii and Bacillus mycoides. Biocontrol Sci. Technol. 12:625-630.

9. Guetsky, R., Shtienberg, D., Elad, Y., Fischer, E., and Dinoor, A. 2002. Improving biological control by combining biocontrol agents each with several mechanisms of disease suppression. Phytopathology 92:976-985.

10. Hamback, P. A., Bjorkman, M., and Hopkins, R. J. 2010. Patch size effects are more important than genetic diversity for plant-herbivore interactions in Brassica crops. Ecol. Entomol. 35:299-306.

11. Janisiewicz, W. J., and Bors, B. 1995. Development of a microbial community of bacterial and yeast antagonists to control wound-invading postharvest pathogens of fruits. Appl. Environ. Microbiol. 61:3261-3267.
12. Jeger, M. J., Jeffries, P., Elad, Y., and Xu, X. M. 2009. A generic theoretical model for biological control of foliar plant diseases. J. Theor. Biol. 256:201-214.

13. Johnson, K. B. 2010. Pathogen refuge: A key to understanding biological control. Annu. Rev. Phytopathol. 48:141-160.

14. Le Floch, G., Tambong, J., Vallance, J., Tirilly, Y., Levesque, A., and Rey, P. 2007. Rhizosphere persistence of three Pythium oligandrum strains in tomato soilless culture assessed by DNA macroarray and real-time PCR. FEMS Microbiol. Ecol. 61:317-326.

15. Le Floch, G., Vallance, J., Benhamou, N., and Rey, P. 2009. Combining the oomycete Pythium oligandrum with two other antagonistic fungi: Root relationships and tomato grey mold biocontrol. Biol. Control 50:288-298

16. Meentemeyer, R. K., Haas, S. E., and Vaclavik, T. 2012. Landscape epidemiology of emerging infectious diseases in natural and humanaltered ecosystems. Annu. Rev. Phytopathol. 50:379-402.

17. Mundt, C. C., Sackett, K. E., and Wallace, L. D. 2011. Landscape heterogeneity and disease spread: Experimental approaches with a plant pathogen. Ecol. Appl. 21:321-328.

18. Paulitz, T. C., and Belanger, R. R. 2001. Biological control in greenhouse systems. Annu. Rev. Phytopathol. 39:103-133.

19. Pierson, E. A., and Weller, D. M. 1994. Use of mixtures of fluorescent pseudomonads to suppress take-all and improve the growth of wheat. Phytopathology 84:940-947.

20. Roberts, D. P., Lohrke, S. M., Meyer, S. L. F., Buyer, J. S., Bowers, J. H., Baker, C. J., Li, W., de Souza, J. T., Lewis, J. A., and Chung, S. 2005. Biocontrol agents applied individually and in combination for suppression of soilborne diseases of cucumber. Crop Prot. 24:141-155.

21. Tscharntke, T., Bommarco, R., Clough, Y., Crist, T. O., Kleijn, D., Rand, T. A., Tylianakis, J. M., van Nouhuys, S., and Vidal, S. 2007. Conservation biological control and enemy diversity on a landscape scale. Biol. Control 43:294-309.

22. With, K. A., Pavuk, D. M., Worchuck, J. L., Oates, R. K., and Fisher, J. L. 2002. Threshold effects of landscape structure on biological control in agroecosystems. Ecol. Appl. 12:52-65.

23. Xu, X.-M., Jeffries, P., Pautasso, M., and Jeger, M. 2011. Combined use of biocontrol agents to manage plant diseases in theory and practice: A review. Phytopathology 101:1024-1031.

24. Xu, X.-M., Jeffries, P., Pautasso, M., and Jeger, M. J. 2011. A numerical study of combined use of two biocontrol agents with different biocontrol mechanisms in controlling foliar pathogens. Phytopathology 101:10321044.

25. Xu, X.-M., and Jeger, M. J. 2013. Combined use of two biocontrol agents with different biocontrol mechanisms most likely results in less than expected efficacy in controlling foliar pathogens under fluctuating conditions: A modeling study. Phytopathology 103:108-116.

26. Xu, X.-M., Salama, N., Jeffries, P., and Jeger, M. J. 2010. Numerical studies of biocontrol efficacies of foliar plant pathogens in relation to the characteristics of a biocontrol agent. Phytopathology 100:814-821.

27. Yobo, K. S., Laing, M. D., and Hunter, C. H. 2010. Application of selected biological control agents in conjunction with tolclofos-methyl for the control of damping-off caused by Rhizoctonia solani. Afr. J. Biotechnol. 9:1789-1796. 